\title{
Ageing of Adhesive Joints for Façade Applications - Comparison of Artificial and Real Weathering Conditions
}

\author{
Barbora Nečasová ${ }^{1, *}$, Pavel Liška ${ }^{1}$, and Michal Novotný ${ }^{1}$ \\ ${ }^{1}$ Brno University of Technology, Veveři 331/95, 60200 Brno, Czech Republic
}

\begin{abstract}
Currently, the adhesive joints are more and more regularly used as an alternative to mechanical joints. They often offer a more suitable, durable and faster possibility. They provide a solution with a more even distribution of stresses in the joint, which consequently allows an increase in the rigidity of the structure and allows it to withstand even heavy loads. High - strength flexible adhesive systems enable the implementation of effective structural joints, and although several decades have passed since the certification and installation of the first façade system with bonded anchoring, even today it is not a matter that is properly grounded in technical standards. A list of specific test procedures on whose basis the behaviour of the joint over the next 40 years could be predicted does not exist. On the basis of these facts, the authors have decided to subject the test samples both to laboratory measurements, where two methods were selected, as well as to expose them to the effects of real weather conditions for a 3-year period. Comparison of the real environment influence results and the methods of artificial aging showed that the selected conditioning methods adequately simulate the effects of weather conditions.
\end{abstract}

\section{Introduction}

The issue of bonding has accompanied humanity throughout its history, yet the greatest development in this area occurred in the last century. Until the beginning of the 20th century, all adhesives used until then were completely natural. Only with the end of the industrial revolution came the first adhesive based on synthetic polymers. Nowadays, it is almost impossible to find a product commonly used in households, industry, transportation, or anywhere else that does not contain adhesive in any form [1].

Currently, bonded joints are becoming increasingly frequent alternatives to mechanical joints. They often offer more convenient, more durable and faster solution. They provide solution with a more even distribution of stresses in the joint [1-3], [4], which consequently increases the toughness of the structure and the transfer of large loads. At the same time, they enable a reduction in the weight of the whole structure, which is often associated with a significant reduction in expenses. The polymeric nature of used adhesives also, to a certain extent, provides for vibration and shock damping, which affect the life and fatigue strength

\footnotetext{
*Corresponding author: necasova.b@,fce.vutbr.cz
} 
of joints. Other advantages include high flexibility of bonded joints, which enables the elimination of deformations resulting from joining materials with different thermal or moisture expansion.

As was already mentioned, bonding has been a popular alternative to mechanical anchoring in recent decades, and its main features were outlined in the previous paragraph. High-strength adhesive/assembly systems allow the realization of effective structural joints, and although the certification and installation of the first façade system with bonded joints was executed several decades ago [5], it is not a technique that is properly rooted in technical standards [6-8]. A good example of a contrasting approach is the engineering industry, where bonding is widely used, for example, in the aviation and automotive industries. Testing procedures for the automotive industry are therefore very often applied even in the field of building testing. The most common methods used to determine the mechanical properties of materials are tensile tests. In such case, test samples are subjected to tensile stress in different directions or at different angles [1-3], [9]. These methods are static when, by applying a uniform load to the test body, they can define properties such as resistance to deformation or deformation properties of the tested material.

In addition to the aforementioned, bonded joints must be designed to withstand not only mechanical stress, but also environmental influences that have a major influence on the life of the joint [5-6], [10-12]. Joint strength is affected by a number of elementary influences, but above all these are temperature, humidity and wind, or more precisely weather conditions. If it is assumed that the bonded joint will be exposed to these influences, it is always appropriate to include test procedures that simulate such conditions, and only then is it appropriate to carry out standardized destructive measurements. Even in the case of simulation of environmental aging, it is possible to proceed according to standardized methods [13-15]. However, these consider and compare only the effects of artificial aging, not the impact of real environment and climate. These are mostly methods prescribing the monitoring of the influence of only one or two parameters, which is almost impossible in a real situation. Machalická and Wolf agree [10-11], that a test procedure that would prescribe a combination of more than two environmental influences does not exist, although for example combinations of humidity, temperature and UV radiation influences are quite common in real environment.

The aim of the presented paper is to compare the influence of standardized methods simulating the real conditions of the outdoor environment with the influence of the actual environment on the strength and life of the bonded joint.

\section{Methodology}

The presented paper deals with bonded joints, which are the anchoring element of façade cladding. The chosen material characteristics of the tested adhesive systems are shown in Table 1 .

Table 1. Mechanical properties of selected adhesives.

\begin{tabular}{|l|c|c|c|}
\hline Property/Adhesive & Type I & Type II & Type III \\
\hline Polymer base & \multicolumn{2}{|c|}{ Polyurethane } & MS Polymer \\
\hline Density $\left[\mathrm{kg} / \mathrm{m}^{3}\right]$ & 1,180 & 1,200 & 1,500 \\
\hline Tear Strength at Break $\left[\mathrm{N} / \mathrm{mm}^{2}\right]$ & 2.50 & 9.00 & 1.80 \\
\hline Shear strength $\left[\mathrm{N} / \mathrm{mm}^{2}\right]$ & 2.00 & 5.50 & 2.25 \\
\hline Service Temperature $\left[{ }^{\circ} \mathrm{C}\right]$ & -40 up to +90 & -40 up to +100 & -40 up to +90 \\
\hline
\end{tabular}


The adhesive system consists of several components, namely a cleaner for removing dirt and grease; a primer to improve adhesion of the bonded surface; an adhesive (only onecomponent polymer-based adhesives hardening with atmospheric moisture were tested here) and mounting tapes that ensure the thickness of the adhesive layer and bear the façade cladding until the adhesive is sufficiently cured.

As façade is the identifying element of each building, it was important from the beginning of the research to make a selection of a suitable material and define its material characteristics. The choice of materials for the purpose of this paper has been greatly simplified since it is only focused on wood and wood-based materials. A Siberian larch (Larix sibirica) façade cladding and a wood plastic composite cladding (hereinafter WPC) was selected.

The next step was to select a suitable test method, the outputs of which are relevant to the chosen structure type. In order to determine the basic physical and mechanical properties of the selected bonded assemblies, a test method to determine adhesion was adapted, i.e. the adhesion of the individual layers of the assembly, which is used to determine the adhesion of the surface treatment to the base [16]. This method is defined by the technical standard ČSN 732577 . The test sample consist of two elements, a cladding of a square section $1=100.0$ $\mathrm{mm}$ and a bearing substructure, also of a square section $1=50.0 \mathrm{~mm}$, which is completely covered with the adhesive. The size of the tested bonded area is therefore $2,500 \mathrm{~mm}^{2}$. The elements are joined together with a selected mounting adhesive with the thickness of $3 \mathrm{~mm}$. The method determines the minimum number of 6 test pieces.

Tensile tests of all test samples to determine strength and relative elongation of the joint after conditioning, see below, were performed on a Heckert FP 10/1 mechanical press. Load speed was set at $5 \mathrm{~mm} / \mathrm{min}$. The deformation of the test samples was recorded using an HBM 1-WA/100 MM-T inductive displacement transducer located on the crosspiece of the tearing press. Testing was carried out at a temperature of $(20 \pm 5){ }^{\circ} \mathrm{C}$ and relative humidity of $(50 \pm$ 20) \%. Test samples were stored well in advance in the test room to allow for their acclimatization.

\subsection{Artificial Weathering}

Façades are naturally exposed to weather conditions, so this paper is focused on selecting methods that simulate these influences. The frost resistance test of the surface treatment of building structures according to ČSN 732579 was chosen. The core of this conditioning method is to alternately freeze and defrost test samples in 15 cycles [17]. The method consists of two parts. In the first step, test samples are immersed in a water bath $(20 \pm 3){ }^{\circ} \mathrm{C}$ for 6 hours. Subsequently, they are removed from the bath and stored in a freezer $(-20 \pm 2){ }^{\circ} \mathrm{C}$ for 18 hours. The storage time in the water and freezer compartment, i.e. 24 hours, is 1 cycle.

The second method of conditioning was the resistance test of the surface treatment of building structures to sudden temperature changes according to ČSN 732581 . The object of the selected conditioning method is alternate heating of the tested samples by infrared lamps and their subsequent cooling in a water bath in 25 cycles [18]. One cycle consists in heating of the bonded joint to a temperature of $(70 \pm 3){ }^{\circ} \mathrm{C}$ and subsequent cooling to a temperature of $(20 \pm 2){ }^{\circ} \mathrm{C}$. This is a temperature that is quite realistic as measured on the test panel, see chapter Analysis. After completion of the conditioning, samples were left for 14 days in a temperature of $(20 \pm 5){ }^{\circ} \mathrm{C}$ and humidity of $(50 \pm 5) \%$.

The selected conditioning methods should suitably substitute the real weather cycles, but only the comparison of the results can confirm this assumption. 


\subsection{Real Weathering Conditions}

Testing of bonded façade joints in a real environment is a non-standardized method. In order to verify the influence of the environment on the strength of the joint, a test panels were made. The test panel geometry was $550 \times 1100 \mathrm{~mm}$. Two variants of façade panels were made, one with Siberian larch façade planks and one with WPC cladding. The load-bearing structure was made of planed and impregnated spruce construction battens of strength class C22 with a 25/45 mm cross section. The axial distance of the studs was $500 \mathrm{~mm}$. The test panels were manufactured according to the adhesive systems manufacturer's instructions, including the application of the mounting tape.

The panels of the chosen combinations of test materials were stored in a real environment. The panels were oriented to the south and stored at an angle of about $60^{\circ}$ for 3 years, i.e. in the period from $06 / 2015$ to $06 / 2018$. Changes in the appearance of the face layer of the façade cladding due to natural aging were monitored. Regular checks and monitoring of occurrence of defects were also carried out. After three years, test samples of the dimensions defined above were produced from the panels. However, in this case the bonded area $\mathrm{A}_{\mathrm{ef}}$ was not uniform, as it had to be determined individually for each test sample after the sample was broken.

In order to assess the impact of the real environment, the weather condition analysis was made in the monitored period from the record of a meteorological station. The device, records outdoor temperature, relative humidity, atmospheric pressure, water precipitation and other quantities [19].

\section{Results}

The adhesion $\left(\sigma_{\text {adh }}\right.$ in $\left.\mathrm{N} / \mathrm{mm}^{2}\right)$, observed when failure occurred in the bonded surface, was determined according to the CSN 732577 standard. The tensile stress on the bonded surface was calculated from the ratio of tensile load $\left(\mathrm{F}_{\max }\right.$ in $\left.\mathrm{N}\right)$ in the moment of failure of the bonded sample and the area of the bonded joint $\left(\mathrm{A}_{\mathrm{ef}}\right.$ in $\left.\mathrm{mm}^{2}\right)$.

The elongation of bonded joints was recorded continuously. Elongation of the bonded joint was calculated using the ratio of the increase in the elongation of the bonded joint $(\Delta \mathrm{l}$ in $\mathrm{mm}$ ) caused by tensile stress to the initial length of the bonded joint $\left(1_{0}\right.$ in $\left.\mathrm{mm}\right)$. As only the change in length was monitored during the tests, we can further talk about relative elongation $(\varepsilon$ in \%).

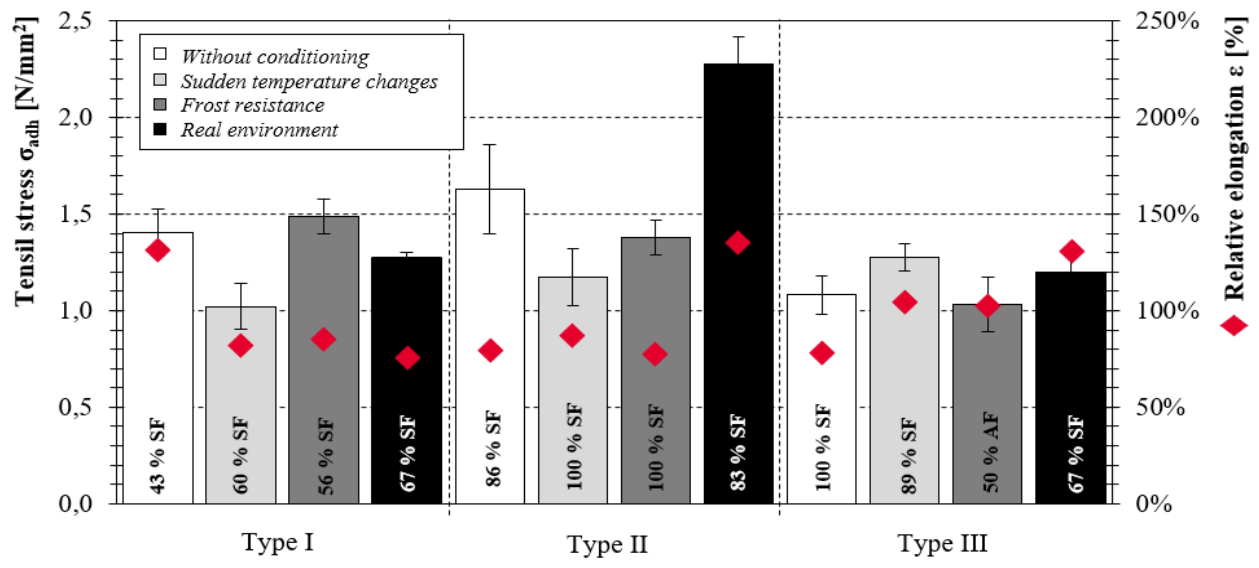

Fig. 1. Comparison of test results - test samples with Siberian larch façade planks - SF is substrate failure (here load-bearing substructure). 


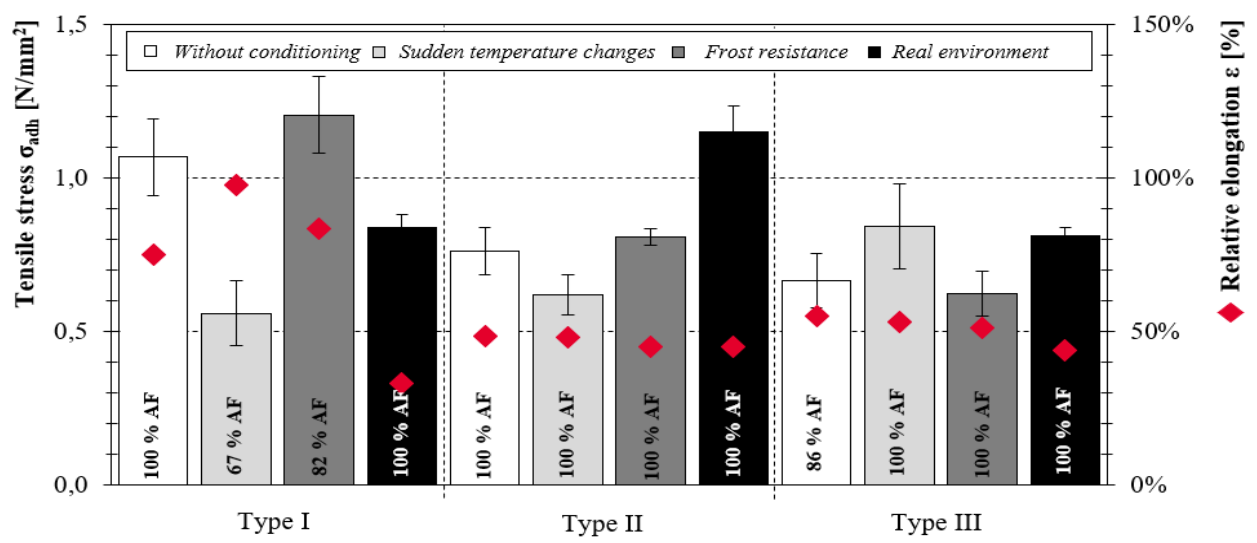

Fig. 2. Comparison of test results - test samples with WPC cladding - AF is adhesive failure (here in the interface of WPC cladding).

The failure modes of test samples were evaluated as well. Recommendations presented in the ČSN ISO 10365 standard [20] and in the international technical standard ASTM D 5573 [21], which defines failure modes in fibre-reinforced composites, were used. For all test samples, failures were evaluated by the same person. All test results are presented in Fig. 1. and Fig. 2., see below.

\section{Analysis}

In general, we can say that for execution of laboratory testing procedures it is important to define the boundary conditions of the controlled environment which enables us to repeat the experiments. However, in the real environment, the implementation of these steps is impossible. Whether it is real weather effects or adherence to technological procedures, the individual applications and measurements will always be different.

The comparison in Fig. 1. show that in the combination with the adhesive Type I, conditioning had an impact, especially on the relative elongation of the joint, it was below $100 \%$ in case of the artificially conditioned samples. As for the adhesive Type II, the effect of the temperature on the joint strength was observed, which decreased by almost $30 \%$. However, it did not affect elongation. Conversely, no impact can be observed in case of the adhesive Type III. While a negative impact of sudden temperature changes on the joint strength were noticed with polyurethane-based adhesive systems (i.e. Type I and Type II), Type I showed almost 50\% strength reduction, while in the combination with Type III (MS polymer), it was quite the opposite, for the joint strength increased by almost $30 \%$. The influence of artificial weathering on the joint elongation is therefore almost negligible.

In the real environment, the recorded data show that the warmest season was the summer months of 2015. A temperature around $36{ }^{\circ} \mathrm{C}$ was monitored. On the other hand, the coldest period was January 2017, temperature around $-17{ }^{\circ} \mathrm{C}$ was observed. Another monitored parameter was ambient humidity, which is expressed by the amount of water vapour in the air. The lowest humidity was measured in February 2018, around $18 \%$, and highest in October 2017, above $98 \%$. Façade panels exposed to real environment influences for three years were regularly monitored and visual surface changes were recorded. Above all, it was the aging of the Siberian larch façade boards. After as soon as 6 months after the installation, a visual change of the Siberian larch cladding was detected. There was a typical grey shade that was more considerable during further checks. This is a typical phenomenon for this type of wood. No change in surface colour was observed in case of the WPC cladding. Wood cladding was also twisting which caused a high tension in the bonded joint. This in some 
boards resulted in the damage of the bonded joint. As for the WPC cladding, a slight bend of individual elements (approx. 2-3 $\mathrm{mm}$ ) was observed, especially during the summer period.

As seen in Fig. 1. and Fig. 2., the greatest strength was achieved in both cases with the Type II. Strengths in case of Type I and Type III are similar. In the case of the Type I in the combination with façade boards, the resulting strengths were greatly affected by excessive twisting of the façade cladding, even though the range of the measurement values does not indicate this. The values of the relative elongation and tensibility of the bonded assemblies presented in Fig. 1. demonstrate very good adhesion of the Type II to the wood cladding. It was detected about $60 \%$ greater elongation of the joint in case of the tested sample group than in the samples tested in the laboratory. On the contrary, in the combination with the Type I, the results were worse than the previous measurements, however, there was a considerable influence of twisting of the cladding. The bonded joint was extremely stressed for more than 2 years, which caused its damage. The results recorded in the combination with the WPC cladding are similar to those of the laboratory measurements. There was adhesion failure and minimal elongation of the joint in all tested combinations. The observed elongations are again comparable to laboratory results, except for the Type I.

A substrate failure of the bearing substructure was the most often noticed failure mode in case of the façade panels with the Siberian larch cladding. This was $72 \%$ of cases under review. This pattern of damage was detected in $85 \%$ of the samples with the adhesive Type II and $67 \%$ of the samples with the Type I and Type III. Cohesive failure was monitored in a case of two samples. It was a damage in the interface between the primer and the adherend of the bearing substructure. In case of two Type I samples the combination of adhesive and cohesive failure was recorded. Especially with the Type III a considerable impact of the tape on the joint strength, which was not used in laboratory tests, was monitored. In some cases, the substructure was delaminated, but the mounting tape was not damaged. The strength of a joint broken like this generally was around $55 \mathrm{~N}$. In the combination with the WPC cladding, I only observed an adhesive way of failure, namely in the interface of primer and the façade cladding. In the combination with the Type III again the effect of the mounting tape on the joint strength was noted. In several cases in the combination with the Type II and Type III systems the adhesive leaked under the mounting tape, which is another issue that can not be tested in laboratory if the technical standards are followed.

\section{Conclusions}

Comparison of the real environment influence results and the methods of artificial aging showed that the selected conditioning methods adequately simulate the effects of weather conditions. As can be seen from the presented results, the measured values are very similar. Only in the combination with the adhesive Type II, higher stress was observed in samples exposed to the real environment. However, only 5 test samples were tested there, so it is not possible to attach much importance to the value. The results monitored in the combination with the WPC cladding confirmed the need for the bonded surface treatment. Even in this combination, values similar to those of the laboratory tests were measured. It is believed that it is possible in this combination to see the mild effect of the environment on the maximum elongation of the bonded joint during break. It is especially true in the combination with the Type I, where the elongation in both claddings was reduced by up to about $61 \%$. To verify this hypothesis, it would be necessary to test a set of more samples. It can be observed from the results that the influence of the real environment on the adhesive Type II is not as significant as the influence of selected conditioning methods. As for both tested claddings, stress on the bonded surface about $60 \%$ higher was measured.

The outputs of the measurements indicate that the selected conditioning methods suitably substitute the effects of the real environment, but the damage of the joint in the real 
environment due to the twisting of the cladding clearly demonstrates that the bonded joint had to withstand a diametrically different stress. It can be assumed that the joint was exposed to a much higher number of cycles than in the case of laboratory tests, which, in the longterm, must have affected its fatigue strength. In some cases, this fact certainly had an effect on premature cohesive failure of the joint. If it was a mechanical joint, the cladding would definitely fall off the façade. Conclusions of the presented experiment also clearly demonstrate that the incorporation of non-standardized procedures is a good way to verify the properties of bonded joints in the real environment.

\section{References}

1. E.M. Petrie, Handbook of Adhesives and Sealants (The McGraw-Hill Companies, Inc., 2007)

2. L.F.M. Da Silva, A. Öchsner, R.D. Adams, Handbook of Adhesion Technology (Springer-Verlag, 2011)

3. S. Ebnesajjad, Adhesives Technology Handbook (William Andrew, 2008)

4. B.R. Burchardt, P.W. Merz, Elastic Bonding and Sealing in Industry (Elsevier Science Ltd, 2006)

5. G. Krüger, R. Schneider, Otto - Graf - Journal, 10, 11 (1999)

6. K. Machalická, M. Vokáč, M. Eliášová, Int. J. Adhes. Adhes., 83 (1), 9 (2018)

7. IJ. J. Straalen et al., Int. J. Adhes. Adhes., 18, (1998)

8. H. Pasternak, Y. Ciupack, Int. J. Adhes. Adhes., 53, 2014

9. P. Cognard, Adhesives and Sealants: Basic Concepts and High-Tech Bonding (ELSEVIER Ltd., 2005)

10. K. Machalická, M. Eliášová, Int. J. Adhes. Adhes., 72, 12 (2017)

11. A.T. Wolf, Durability of building sealants (RILEM Publ., 1999)

12. G. Krüger, G.E. Völkel, Otto - Graf Journal, 10, 8 (1998)

13. ETAG 002. Structural Sealant Glazing Kits. Brusel: European Organisation for Technical Approvals, 2001. Available on http://www.eota.org

14. ČSN EN ISO 9142. Adhesives - Guide to the selection of standard laboratory ageing conditions for testing bonded joints, (Czech institute for standardization, 2007)

15. ČSN EN ISO 11431. Building construction - Jointing products - Determination of adhesion/cohesion properties of sealants after exposure to heat, water and artificial light through glass, (Czech institute for standardization, 2003)

16. $\check{C} S N 732577$. Test for surface finish adhesion of building structures to the base, (Czech institute for standardization, 1981)

17. ČSN 73 2579. Test for frost resistance of surface finish of building structures, (Czech institute for standardization, 1981)

18. ČSN 73 2581. Test for resistance or surface finish of building structures to acute temperature variations, (Czech institute for standardization, 1983)

19. Available on http://www.hvezdarna.cz/?page id=1255

20. ČSN ISO 10365. Adhesives. Designation of main failure patterns. (Czech institute for standardization, 1995)

21. ASTM D 5573. Standard Practice for Classifying Failure Modes in Fiber-ReinforcedPlastic (FRP) Joints (ASTM International, 2005) 